\title{
Simulation of the Electric Field of a Point Source on the Relief Surface
}

\begin{abstract}
B. Mukanova, T. MirgalikyzY*
L.N. Gumilyov Eurasian National University, Faculty of Information Technologies, Astana, Kazakhstan

This research examines the numerical solutions to the direct problem of electrical impedance tomography for two-dimensional and three-dimensional media, taking into count the surface relief on the basis of the integral equations method. The results of the numerical modeling identify a distortion effect for vertical electrical sounding curves caused by relief surface and the apparent resistivity function along the relief surface is obtained.
\end{abstract}

DOI: 10.12693/APhysPolA.128.B-142

PACS: 02.70.-c, 93.85.-q

\section{Introduction}

Accounting the impact of the surface relief during the solution of direct problem of electrical prospecting is today's important issue. Currently, the main methods used in practice for solving direct and inverse problems are difference methods (finite differences method and finite elements method) [1,2]. Within these methods several methods of accounting relief influence were developed.

In the method of finite differences two basic algorithms with surface topography observations were developed $[3,4]$. Obviously, all the circuits of the cell which have a rectangular shape, comprise geometric error in consideration of the effect of the relief. It is possible to reduce the error only by increasing the number of cells, which leads to a significant increase in computational cost [5].

The finite element method in the addition to the direct problem solution of direct current methods is rapidly developing $[6,7]$.

Versatility, flexibility and number of computational advantages of grid methods defined their widespread application in many areas of numerical simulations, and, in particular, in the methods of direct current. However, when specifying the model with the relief most of the cell configurations of the grid somehow repeated the configuration of the relief. This may add some errors in the solution of both direct and, most importantly, the inverse problems. Therefore, to control the operation of difference methods it is appropriate to apply other methods based on the different ideas and also allows to solve the direct problems of the resistivity method. The method of integral equations, which has a simple physical meaning and was well-established during the simulation of methods of resistivity, was chosen as the part of this work [8-11].

\section{Methods}

One way to study the structure of the geo-electric section is a vertical electrical sounding method, which can

*corresponding author; e-mail: m_t85@mail.ru be considered as a modification of electrical impedance tomography. In the method of electrical tomography, the obtained results (geo-electric section) strongly depend on the following factors: on geometry of the electrodes' array or relative positions of current and receiver electrodes, on structure of geo-ecologic section or, in other words, distribution of resistivity $(\rho(x, y, z))$ and on Earth surface relief. The last factor complicates the task of solving the inverse problem of finding the distribution of $\rho(x, y, z)$ resistivity.

Conducting interpretation without the influence of topography can cause non-existent anomalies on sections. Thus, taking account of the relief profile of observations is a rather complicated problem.

There are algorithms of solving direct and inverse problems of geophysics in the basis of programs of inversion. Today we know that mostly often used method of solving direct problems is finite differences and finite element methods. They are useful for a big amount of practical aims, but they have several disadvantages [5]. In particular, these are: low accuracy of field's first derivatives computation, and subsequently computations of apparent resistivity, problem of creating the grid, adapted to complicated form of the boundary of a computation domain, problem of producing of artificial boundary conditions on boundaries of computation domain, problem of singularity, related to the function of the source and to the form of the relief.

On the other hand, purposes of geophysics have high requirements on accuracy and speed of field computations. As an alternative method of solving the direct problems, satisfying both of these requirements is the method of integral equations $[8,9]$. The idea of this method is to provide the electric field as a sum of the primary field (generated by current electrodes, in this case by one electrode) and the field of secondary charges (occur when the electric current flows in points of homogeneity violation of the medium and on the surface of the medium). Field computation problem is reduced to the system of integral equations on density of secondary sources, induced on contact surfaces of conductive media and on relief surface of the medium. 


\section{Mathematical model and formulation of the problem}

We consider the mathematical model of a direct current sounding method for medium with constant resistivity with any relief on the surface. It is known from th Maxwell equations that the stationary field electrostatic potential at points in the medium satisfies the differential Laplace Eq. (1):

$$
\frac{\partial^{2} \varphi}{\partial x^{2}}+\frac{\partial^{2} \varphi}{\partial y^{2}}+\frac{\partial^{2} \varphi}{\partial z^{2}}=0
$$

The condition of decreasing the potential at infinity $\varphi(\infty)=0$, and the condition on boundary of earth-air $\left.\frac{\partial \varphi}{\partial z}\right|_{z=0}=0$ must be satisfied.

It is required to calculate the apparent resistivity function along the surface defined by the relation (2):

$$
\rho_{k}=K \frac{\partial \varphi}{\partial r} \frac{1}{I}, \quad K=\frac{2 \pi A M A N}{M N},
$$

where $I$ is current source electrode, $K$ is geometrical factor of the electrode array.

\section{Numerical results}

To calculate the field on the relief surface we assigned some distribution of current density on the surface, which compensate for it so as to satisfy the physical boundary condition (3):

$$
\left.\sigma \frac{\partial \varphi}{\partial n}\right|_{\Gamma}=-\left.\sigma \frac{\partial U_{0}}{\partial n}\right|_{\Gamma}
$$

Here, $\sigma$ is the layer electrical conductivity, and $U_{0}$ is the potential of a source electrode. For distribution of current densities on the relief surface we used a grid with a logarithmic expanding scale by radial, adapted to the position of the source electrode.

We have built the integral equation for distribution of secondary sources, taking into account the given boundary condition and the Green formula. The following is the integral Eq. (4) for distribution of secondary sources at points of $M$ on the medium surface, formed under the influence of a single point current source, located at some point on the surface. Under the integrand there calculates the mutual influence of all other sources on the source of $M$. From the integral equation it is required to calculate the current density of secondary source, $I_{\mathrm{S}}(P)$.

$$
\begin{aligned}
& I_{\mathrm{S}}(M)=-2 \sigma\left(\iint_{\Gamma} \frac{\partial G(P, M)}{\partial n} I_{\mathrm{S}}(P) \mathrm{d} S_{\mathrm{P}}+E_{0}(M)\right), \\
& \frac{\partial G(P, M)}{\partial n}=\frac{1}{\sigma 4 \pi} \frac{\boldsymbol{r}_{\mathrm{PM}}}{r_{\mathrm{PM}}^{3}} \mathbf{1},
\end{aligned}
$$

where $I_{\mathrm{S}}(M)$ is the current density of secondary source at point of $M$ on the surface $\Gamma, E_{0}(M)$ is the electric field from a primary source at this point $M, G(P, M)$ is the Green function, $I_{\mathrm{S}}(P)$ is the current density of secondary source at point of $P, \mathrm{~d} S_{\mathrm{P}}$ is the surface area where the source $P$ is. Equation (4) expresses the physical fact that at the points of $M$ of the surface the total current is zero, except the current source electrode.
In Fig. 1 there is plotted the density of secondary sources, obtained by solving the integral Eq. (4).

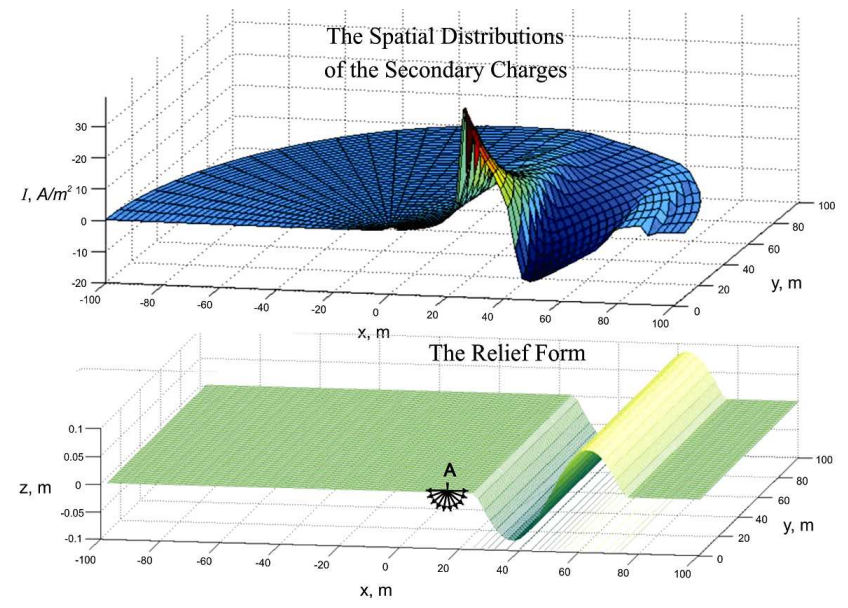

Fig. 1. Solution of the system of integral equations: the spatial distributions of the secondary charges and the relief form.

After calculating the distribution of secondary sources, the values of the potentials on the surface are calculated (5):

$$
\begin{aligned}
& \frac{\partial \varphi}{\partial r}(M)=U_{0}(M)+\iint_{\Gamma} \frac{I_{\mathrm{S}}(P)}{\sigma 4 \pi} \frac{1}{\left|r_{\mathrm{PM}}\right|} \mathrm{d} S_{\mathrm{P}}, \\
& U_{0}(M)=\frac{I_{\mathrm{S}}(M)}{\sigma 2 \pi} \frac{1}{\left|r_{\mathrm{PM}}\right|} .
\end{aligned}
$$

$U_{0}(M)$ is the potential of a source electrode.

Figure 2 shows the solution of the problem - the apparent resistivity function along the relief surface.

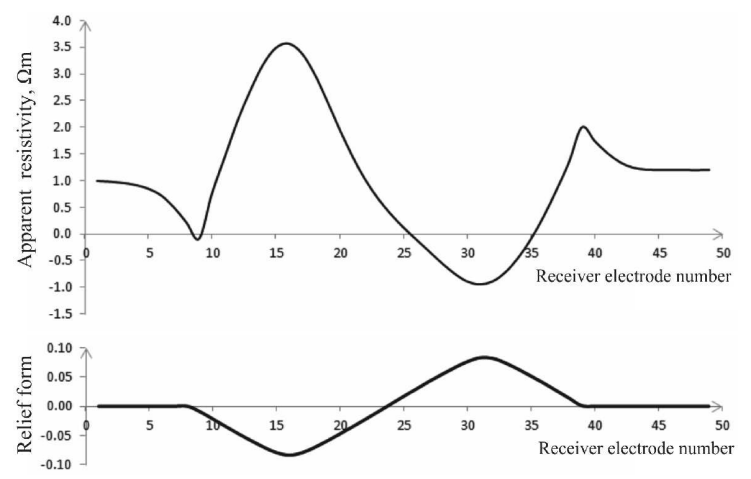

Fig. 2. The apparent resistivity and the relief form.

\section{Conclusion}

The results of the numerical modeling identified a distortion effect for curves of vertical electrical sounding caused by relief surface. The results will encourage increase of work quality and development of computer technologies used in geophysics. In common, increase of the quality of interpretation, due to more accurate calculation of the medium conditions, increases efficiency of geophysical researches. 


\section{Acknowledgments}

The work was supported by the Ministry of Education and Science of the Republic of Kazakhstan, grant number is $46-12.02 .2015$.

\section{References}

[1] J.H. Coggon, Geophysics 36, 132 (1971).

[2] M.H. Loke, R.D. Barker, Geophys. Prospect. 44, 131 (1996).

[3] I. Demirci, E. Erdogan, M.E. Candasayar, Geophysics 77, 67 (2012).

[4] S. Penz, H. Chauris, D. Donno, C. Mehl, Geophys. J. Int. 194, 1486 (2013).
[5] M. Orunkhanov, B. Mukanova, B. Sarbassova, in: Joint issue of Comp. Techn. 9 and Bulletin of KazNU 3/42: Proc. Int. Conf. "Computational and Informational Technologies for Science, Engineering and Education", Almaty (Kazakhstan), 2004 (in Russian).

[6] R.C. Fox, G.W. Hohmann, T.J. Killpack, L. Rijo, Geophysics 45, 75 (1980).

[7] T. Gunther, C. Rucker, Boundless Electrical Resistivity Tomography. BERT - the User Tutorial, 2011.

[8] G.W. Hohmann, Geophysics 40, 309 (1975).

[9] M. Orunkhanov, B. Mukanova, Adv. High Perform. Comput. Computat. Sci. 93, 15 (2006). 